\title{
SHORT GOMMUNIGATIONS
}

\section{ON THE SUFFIGIENT STATISTIGS FOR STATIONARY GAUSSIAN RANDOM PROGESSES}

\section{ARATO}

(Translated by N. Greenleaf)

This note is devoted to finding sufficient statistics for a stationary Gaussian random process with discrete time in the most simple case, where the spectral density is a rational function with respect to $e^{i \lambda}$. In the case where the stationary Gaussian process $\xi(t)$ has spectral density of the form

$$
f_{\xi}(\lambda)=\frac{\sigma_{\varepsilon}^{2}}{2 \pi \mid 1+\alpha_{1} e^{-i \lambda}+\cdots+\alpha_{p} e^{-\left.i \lambda_{p}\right|^{2}}},
$$

it satisfies the difference equation

$$
\xi(t)+\alpha_{1} \xi(t-1)+\cdots+\alpha_{p} \xi(t-p)=\varepsilon(t),
$$

where $\sigma_{\varepsilon}^{2}=\mathbf{M} \varepsilon^{2}(t)$ (Doob [1], page 450), $\varepsilon(t)$ are mutually independent random variables, and the mathematical expectation $\mathbf{M} \xi(t)=0$. It turns out that the number of functions of the sample values $x_{1}, \cdots, x_{N}$, forming necessary and sufficient statistics for the family of distributions with unknown parameters $\alpha_{1}, \cdots, \alpha_{p}$ and $\sigma_{\xi}^{2}=\mathbf{M} \xi^{2}(t)$, is equal to $(p+1)(p+2) / 2$. We shall also show by a simple example that in the general case this is not so.

By the well-known theorem of Dynkin [2], the expressions

$$
\begin{aligned}
\log p \xi(1), \cdots, \xi(N)\left(x_{1},\right. & \left.\cdots, x_{N} ; \alpha_{1}, \cdots, \alpha_{p}, \sigma_{\xi}{ }^{2}\right) \\
& -\log p_{\xi(1)}, \cdots, \xi(N)\left(x_{1}, \cdots, x_{N} ; \alpha_{1}{ }^{0}, \cdots, \alpha_{p}{ }^{0}, \sigma_{\xi}{ }^{{ }^{0}}\right)
\end{aligned}
$$

form a necessary and sufficient statistics for the family of distributions $p_{\xi(1)}, \cdots, \xi(N)\left(y_{1}, \cdots, y_{N}\right.$; $\left.\alpha_{1}, \cdots, \alpha_{p}, \sigma_{\xi}^{2}\right)$, where $p_{\xi(1)}, \cdots, \xi(N)\left(y_{1}, \cdots, y_{N} ; \alpha_{1}, \cdots, \alpha_{p}, \sigma_{\xi}^{2}\right)$ is the joint density of the probability of the variables $\xi(1), \cdots, \xi(N) ; x_{1}, \cdots, x_{N}$ is the sample, and $\alpha_{1}^{0}, \cdots, \alpha_{p}{ }^{0}, \sigma_{\xi}{ }^{2^{0}}$ are fixed values of the parameters. From this theorem easily follows the following

Theorem. If the Gaussian stationary process $\xi(t)$ has spectral density of the form $(1)$, then the system

$$
\left.\begin{array}{rl}
\left(\sum_{i=p+1}^{N-p} x_{i}{ }^{2}, \sum_{i=p+1}^{N-p+1} x_{i} x_{i-1}, \cdots,\right. & \sum_{i=p+1}^{N} x_{i} x_{i-p}, x_{1}^{2}+x_{N}{ }^{2}, x_{1} x_{2}+x_{N} x_{N-1}, \\
\cdots, x_{1} x_{p}+x_{N} x_{N-p+1}, x_{2}^{2}+x_{N-1}^{2}, \cdots, x_{2} x_{p}+x_{N-1} x_{N-p+1}, \ldots, x_{p}^{2}+x_{N-p+1}^{2}
\end{array}\right)
$$

of functions of the sample $x_{1}, \cdots, x_{N}$ forms a necessary and sufficient statistics.

To prove this we calculate the joint density of the probability of these variables. Multiplying (2) by $\xi(t-1), \cdots, \xi(t-p)$ and taking the mathematical expectation, we obtain the following system of linear equations for determining the correlation coefficient

$$
\begin{gathered}
\lambda_{i}=\frac{\mathbf{M} \xi(t) \xi(t-i)}{\sigma_{\xi}^{2}}, \\
\alpha_{1}+\alpha_{2} \lambda_{1}+\cdots+\alpha_{p} \lambda_{p-1}+\lambda_{1}=0, \\
\alpha_{1} \lambda_{1}+\alpha_{2}+\cdots+\alpha_{p} \lambda_{p-2}+\lambda_{2}=0 \\
\cdots \cdots+\cdots+\cdots+\cdots+\alpha_{p}+\lambda_{p}=0 . \\
\alpha_{1} \lambda_{p-1}+\alpha_{2} \lambda_{p-2}+\cdots+\cdots+\alpha
\end{gathered}
$$$$
i=1,2, \cdots, p,
$$

In addition, we have

$$
\sigma_{\xi}^{2}+\sigma_{\xi}^{2}\left(\alpha_{1} \lambda_{1}+\cdots+\alpha_{p} \lambda_{p}\right)=\sigma_{\varepsilon}^{2}
$$


We let $A\left(\alpha_{1}, \cdots, \alpha_{p}\right)=\sigma_{\varepsilon}^{2} / \sigma_{\xi}^{2}$, and denote by $B\left(\alpha_{1}, \cdots, \alpha_{p}\right)$ the determinant of the covariance matrix of the random variables $\xi(1), \cdots, \xi(p)$ and, finally, by $\left\{b_{i j}^{*}\right\}_{1}{ }^{p}$ the inverse of the covariance matrix. Then the probability densities will have the form

$$
\begin{aligned}
p_{\xi(1), \cdots, \xi(N)}\left(y_{1}, \cdots, y_{N}\right)=p_{\xi(1), \cdots, \xi(p)}\left(y_{1}, \cdots, y_{p}\right) \cdot p_{\varepsilon(p+1)}\left(y_{p+1}+\alpha_{1} y_{p}+\cdots+\alpha_{p} y_{1}\right) \\
\cdots p_{\varepsilon(N)}\left(y_{N}+\alpha_{1} y_{N-1}+\cdots+\alpha_{p} y_{N-p}\right) \\
=(2 \pi)^{-N / 2} \sigma_{\xi}^{-N} B\left(\alpha_{1}, \cdots, \alpha_{p}\right)^{-1 / 2} A\left(\alpha_{1}, \cdots, \alpha_{p}\right)^{-(N-p) / 2} \exp \left\{-\frac{1}{2} \sum_{i, j=1}^{p} b_{i j}^{*} y_{i} y_{j}\right. \\
\left.-\frac{1}{2 \sigma_{\xi}^{2} A} \sum_{i=p+1}^{N}\left(y_{i}+\alpha_{1} y_{i-1}+\cdots+\alpha_{p} y_{i-p}\right)^{2}\right\} .
\end{aligned}
$$

We note that in mapping $\xi(1), \cdots, \xi(N)$ onto $\xi(1), \cdots, \xi(p), \varepsilon(p+1), \cdots, \varepsilon(N)$, the Jacobian is equal to one, and that the logarithm of the probability density has, up to a constant, the form (taking into account that the covariance matrix, and therefore also the matrix of the quantities $\xi(1), \cdots, \xi(N)$ which is inverse to it, is symmetric about its diagonal):

$$
\begin{gathered}
\frac{1+\alpha_{1}^{2}+\cdots+\alpha_{p}^{2}}{2 \sigma_{\xi}^{2} A} \sum_{i=p+1}^{N-p} y_{i}^{2}+\frac{\alpha_{1}+\alpha_{1} \alpha_{2}+\cdots+\alpha_{p-1} \alpha_{p}}{\sigma_{\xi}^{2} A} \sum_{i=p+1}^{N-p+1} y_{i} y_{i-1}+\cdots \\
+\frac{\alpha_{p}}{\sigma_{\xi}^{2} A} \sum_{i=p+1}^{N} y_{i} y_{i-p}+\left(y_{1}^{2}+y_{N}^{2}\right) \frac{1}{2 \sigma_{\xi}^{2} A}+\left(y_{1} y_{2}+y_{N} y_{N-1}\right) \frac{\alpha_{1}}{\sigma_{\xi}^{2} A}+\cdots \\
+\left(y_{1} y_{p}+y_{N} y_{N-p+1}\right) \frac{\alpha_{p-1}}{\sigma_{\xi}^{2} A}+\left(y_{2}^{2}+y_{N-1}^{2}\right) \frac{1+\alpha_{1}^{2}}{2 \sigma_{\xi}^{2} A} \\
+\left(y_{2} y_{3}+y_{N-1} y_{N-2}\right) \frac{\alpha_{1}+\alpha_{1} \alpha_{2}}{\sigma_{\xi}^{2} A}+\cdots+\left(y_{2} y_{p}+y_{N-1} y_{N-p+1}\right) \frac{\alpha_{p-2}+\alpha_{1} \alpha_{p-1}}{\sigma_{\xi}^{2} A} \\
+\left(y_{p}^{2}+y_{N-p+1}^{2}\right) \frac{1+\alpha_{1}^{2}+\cdots+\alpha_{p-1}^{2}}{2 \cdot \sigma_{\xi}^{2} \cdot A}
\end{gathered}
$$

The proof of the assertion easily follows from this. In the same way one determines sufficient statistics in the case where the mathematical expectation is unknown.

The following example shows that in the general case the assertion of the theorem does not hold. Consider the stationary Gaussian process

$$
\xi(t)=a_{0} \varepsilon(t)+a_{1} \varepsilon(t-1),
$$

where $\varepsilon(t)$ are mutually independent Gaussian random variables. We shall show that in this case there do not exist functions of the sample $x_{1}, \cdots, x_{N}$, whose number is less than $N$, such that they form sufficient statistics for $a_{0}, a_{1}, \sigma_{\xi}^{2}=\mathbf{M} \xi^{2}(t)$ (we assume here that $\mathbf{M} \xi(t)=0$ ).

From equation (5) it follows that

$$
\begin{aligned}
\sigma_{\xi}{ }^{2}=\mathbf{M} \xi^{2}(t)= & \left(a_{0}{ }^{2}+a_{1}{ }^{2}\right) \mathbf{M} \varepsilon^{2}(t), \quad \rho=\frac{\mathbf{M} \xi(t) \xi(t-1)}{\sigma_{\xi}{ }^{2}}=\frac{a_{0} a_{1}}{a_{0}{ }^{2}+a_{\mathbf{1}}{ }^{2}}, \\
& \mathbf{M} \xi(t) \xi(t-\tau)=0, \quad \text { if } \quad|\tau|>1 .
\end{aligned}
$$

The joint probability density of the variables $\xi(1), \cdots, \xi(N)$ has the form:

$$
p_{\xi(1)}, \cdots, \xi(N)\left(y_{1}, \cdots, y_{N}\right)=\sigma_{\xi}^{-N} \cdot(2 \pi)^{-N / 2} \cdot\left|B_{N}\right|^{-1 / 2} \exp \left\{-\frac{1}{2 \sigma_{\xi}^{2}} \sum_{i, j=1}^{N} b_{i j}^{*} y_{i} y_{j}\right\},
$$

where $\left|B_{N}\right|=\operatorname{det} B_{N}$ and $B_{N}^{-1}=\left\{b_{i j}^{*}\right\}_{1}^{N}$ is the inverse matrix of the correlation matrix $B_{N}$ of the process $\xi(t)$. It is easily calculated that

$$
b_{i j}^{*}=(-1)^{j-i} \rho^{j-i}\left|B_{i-1}\right|\left|B_{N-j}\right| \frac{1}{\left|B_{N}\right|}
$$

for $i<j$ and $\left|B_{i}\right|=\left(u_{1}^{i+1}-u_{\mathbf{2}}^{i+1}\right) /\left(u_{1}-u_{2}\right)$ (the inverse matrix is assumed to be symmetric about the diagonal). It should be noted that $\left|B_{N}\right|$ satisfies the difference equation $\left|B_{N}\right|=\left|B_{N-1}\right|-\rho^{2}\left|B_{N-2}\right|$ and $u_{1}$ and $u_{2}$ are solutions of the equations

$$
u^{2}-u+\rho^{2}=0, \text { i.e. } \quad u_{1}=\frac{1+\sqrt{1-4 \rho^{2}}}{2}, \quad u_{2}=\frac{1-\sqrt{1-4 \rho^{2}}}{2} .
$$

Since, further, $b_{i N}^{*}, i=1, \cdots, N$, are independent as functions of $\rho$, it becomes clear (see, for example, 
Dynkin [2], § 2) that there do not exist functions of the samples $x_{1}, \cdots, x_{N}$, less than $N$ in number, which would form sufficient statistics for $a_{0}, a_{1}, \sigma_{\xi}{ }^{2}$.

I consider it a most pleasant duty to express my warm thanks to A. N. Kolmogorov for posing this problem and for advice given me in the writing of this note.

Received by the editors, April 21, 1960

\section{REFERENCES}

[1] J. L. Doob, Stochastic Processes, Wiley, New York, 1953.

[2] E. B. Dynkin, Necessary and sufficient statistics for families of probability distributions, Uspekhi Matem. Nauk, 6, 1951, pp. 68-90. (In Russian.)

\section{ON THE SUFFIGIENT STATISTIGS FOR STATIONARY GAUSSIAN RANDOM PROGESSES}

\section{ARATO $(M O S C O W)$}

(Summary)

We prove that for a stationary Gaussian process with spectral density (1) the number of sufficient statistics is $(p+1)(p+2) / 2$. A simple example shows that in the general case the number of sufficient statistics increases with the number of observations.

\section{GONGERNING A GERTAIN PROBABILITY PROBLEM}

\section{M. ZOLOTAREV}

(Translated by Mario Petrich)

1. Let $\xi_{1}, \xi_{2}, \xi_{3}, \cdots$ be a sequence of independent normally distributed random variables with parameters $(0,1)$ and let

$$
\begin{gathered}
\lambda_{1}^{2}=\lambda_{2}^{2}=\cdots=\lambda_{n_{1}}^{2} \\
\lambda_{n_{1}+1}^{2}=\lambda_{n_{1}+2}^{2}=\cdots=\lambda_{n_{1}+n_{2}}^{2} \\
\cdots \ldots \ldots \ldots \ldots \ldots \ldots \ldots
\end{gathered}
$$

be some sequence of positive numbers such that representatives of separate blocks (we shall denote them by $\left.\sigma_{r}^{2}, r=1,2, \cdots\right)$ form a strictly decreasing sequence and

$$
\sum_{k=1}^{\infty} \lambda_{k}{ }^{2}=\sum_{r=1}^{\infty} n_{r} \sigma_{r}^{2}<\infty .
$$

We construct the random variable $\eta=\Sigma_{k} \lambda_{k}^{2} \xi_{k}^{2}$, which exists, obviously, with probability $\mathbf{1}$. The question is raised about asymptotic behavior $($ as $x \rightarrow \infty)$ of the function $\mathbf{P}\{\eta \geqq x\}=1-F_{\eta}(x)$ and of the density $p_{\eta}(x)$. Some problems of probability and mathematical statistics lead to this $\mathrm{s}^{\text {cheme. }}$

2. In as much as $\eta$ is a non-negative random variable, we can use the apparatus of Laplace transformations, existing in the half plane $\operatorname{Re} s \geqq 0$. It is not hard to convince ourselves that at least in this half plane

$$
\varphi(s)=\mathbf{M} \exp (-s \eta)=\prod_{k}\left(1+2 s \lambda_{k}^{2}\right)^{-1 / 2}=\prod_{r}\left(1+2 s \sigma_{r}^{2}\right)^{-n_{r} / 2} .
$$

By condition (1) this product converges for all complex $s$ with the exception of the points $s_{r}=-1 / 2 \sigma_{r}^{2}, r=1,2, \cdots$.

Consequently, the function $\varphi(s)$ can be continued into the left half plane (with the exception, naturally, of the points $s_{r}$ ), moreover,

a) if all multiplicities of factors in product (2) are even, then $\varphi(s)$ is a meromorphic function with poles of order $n_{r} / 2$ at the points $s_{r}$;

b) if some of the numbers $n_{r}$ are odd, then $\varphi(s)$ is a multiple-valued function with branch points of second order in those points $s_{r}$ for which the numbers $n_{r}$ are odd and poles of order $n_{r} / 2$ in points $s_{r}$ for which these multiplicities are even. 
циента корреляци

$$
\left.\begin{array}{c}
\lambda_{i}=\frac{M \xi(t) \xi(t-i)}{\sigma_{\xi}^{2}}(i=1,2, \ldots, p) \\
\alpha_{1}+\alpha_{2} \lambda_{1}+\ldots+\alpha_{p} \lambda_{p-1}+\lambda_{1}=0, \\
\alpha_{1} \lambda_{1}+\alpha_{2}+\ldots+\alpha_{p} \lambda_{p-2}+\lambda_{2}=0, \\
\ldots \ldots \ldots \ldots \ldots \ldots \ldots \ldots+\alpha_{p}+\lambda_{p}=0 . \\
\alpha_{1} \lambda_{p-1}+\alpha_{2} \lambda_{p-2}+\ldots+
\end{array}\right\}
$$

Кроме того, имеем

$$
\sigma_{\xi}^{2}+\sigma_{\xi}^{2}\left(\alpha_{1} \lambda_{1}+\ldots+\alpha_{p} \lambda_{p}\right)=\sigma_{\varepsilon}^{2}
$$

Обозначим через $A\left(\alpha_{1}, \ldots, \alpha_{p}\right)=\frac{\sigma_{\varepsilon}^{2}}{\sigma_{\xi}^{2}}$, через $B\left(\alpha_{1}, \ldots, \alpha_{p}\right)$ определитель ковариа-

0 ДОСТАТОЧНЫХ СТАТИСТИКАХ СТАЦИОНАРНЫХ

ГАУССОВСКИХ СЛУЧАЙНЫХ ПРОЦЕССОВ

\section{APATO}

Чac. сал в самом простом случае, коггауссовских случайных процессов с дискретным временем в сай омнотель $e^{i \lambda}$. В случае, когда стационарный гауссовский процесс $\xi(t)$ имеет спектральную плотность вида

$$
f_{\xi}(\lambda)=\frac{\sigma_{\varepsilon}^{2}}{2^{\pi}\left|1+\alpha_{1} e^{-i \lambda}+\ldots+\alpha_{p} e^{-i \lambda_{p}}\right|^{2}},
$$

то он удовлетворяет разностному уравнению

$$
\xi(t)+\alpha_{1} \xi(t-1)+\ldots+\alpha_{p} \xi(t-p)=\varepsilon(t),
$$

где $\sigma_{\varepsilon}^{2}=M \varepsilon^{2}(t)$, (Дуб [1], стр. 450), $\varepsilon(t)$ - независимые друг ог друга случайные величины, а математическое ожидание $\mathbf{M} \xi(t)=0$. Оказывается, что число функций от выборочных значений $x_{1}, \ldots, x_{N}$, образующих необходимую и достаточную статистику для семейства распределений с неизвестными параметрами $\alpha_{1}, \ldots, \alpha_{p}$ и $\sigma_{\xi}^{2}=\mathbf{M} \xi^{2}(t)$, равно $\frac{(p+1)(p+2)}{2}$. Мы покажем также на простом примере, что в общем случае это не так.

По известной теореме Е. Б. Дынкина [2] выражение

$$
\begin{aligned}
& \log p_{\xi(1), \ldots, \xi(N)}\left(x_{1}, \ldots, x_{N} ; \alpha_{1}, \ldots, \alpha_{p}, \sigma_{\xi}^{2}\right)- \\
& -\log p_{\xi(1), \ldots, \xi(N)}\left(x_{1}, \ldots, x_{N} ; \alpha_{1}^{0}, \ldots, \alpha_{p}^{0}, \sigma_{\xi}^{2^{0}}\right)
\end{aligned}
$$

является необходимой и достаточной статистикой для семейства распределений $p_{\xi(1), \ldots, \xi(N)}\left(y_{1}, \ldots, y_{N} ; \alpha_{1}, \ldots, \alpha_{p}, \sigma_{\xi}^{2}\right)$, где $p_{\xi(1), \ldots, \xi(N)}\left(y_{1}, \ldots, y_{N} ; \alpha_{1}, \ldots, \alpha_{p}, \sigma_{\xi}^{2}\right)-$ совместиая плотность вероятности величин $\xi(1), \ldots, \xi(N) ; x_{1}, \ldots, x_{N}$ - выборка и $\alpha_{\mathbf{1}}^{0}, \ldots, \alpha_{p}^{0}, \sigma_{\xi}^{2^{0}}-$ фиксированные значения параметров. Из этой теоремы легко вытекает следующая

Теорема. Если гауссовский стациионарный процесс $\xi(t)$ имеет спектральную плотность вида (1), по система

$$
\left(\sum_{i=p+1}^{N-p} x_{i}^{2}, \sum_{i=p+1}^{N-p+1} x_{i} x_{i-1}, \ldots, \sum_{i=p+1}^{N} x_{i} x_{i-p}, x_{1}^{2}+x_{N}^{2}, x_{1} x_{2}+x_{N} x_{N-1}, \ldots\right.
$$

$\left.\ldots, x_{1} x_{p}+x_{N} x_{N-p+1}, x_{2}^{2}+x_{N-1}^{2}, \ldots, x_{2} x_{p}+x_{N-1} x_{N-p+1}, \ldots, x_{p}^{2}+x_{N-p+1}^{2}\right)$

функиий от выборки $x_{1}, \ldots, x_{N}$ образует необходияую и достатоиную статистику. Для доказательства подсчитаем совместную плотность вероятности этих величин. Умножая уравнение $(2)$ на $\xi(t-1), \ldots, \xi(t-p)$ и взяв математическое ожидание, получаем следующую систему линейных уравнений для определения коэФФб- ционной матрицы случайных величин $\xi(1), \ldots, \xi(p)$ и, наконец, через $\left\{b_{i j}^{*}\right\}_{1}^{p}-$ матрицу, обратную к ковариационной матрице. Тогда плотность вероятности будет иметь вид $p_{\xi(1), \ldots, \xi(N)}\left(y_{1}, \ldots, y_{N}\right)=p_{\xi(1), \ldots, \xi(p)}\left(y_{1}, \ldots, y_{p}\right) \cdot p_{\varepsilon(p+1)}\left(y_{p+1}+\alpha_{1} y_{p}+\ldots+\alpha_{p} y_{1}\right) .$. $\cdots p_{\varepsilon(N)}\left(y_{N}+\alpha_{1} y_{N-1}+\ldots+\alpha_{p} y_{N-p}\right)=$

$$
\begin{gathered}
=(2 \pi)^{-N / 2} \sigma_{\xi}^{-N} B\left(\alpha_{1}, \ldots \alpha_{p}\right)^{\sim 1 / 2} A\left(\alpha_{1}, \ldots, \alpha_{p}\right)^{-\frac{N-p}{2}} \exp \left\{-\frac{1}{2} \sum_{i, j=1}^{p} b_{i j}^{*} y_{i} y_{j}-\right. \\
\left.-\frac{1}{2 \sigma_{\xi}^{2} A} \sum_{i=p+1}^{N}\left(y_{i}+\alpha_{1} y_{i-1}+\ldots+\alpha_{p} y_{i-p}\right)^{2}\right\} .
\end{gathered}
$$

Заметим, что при отображении $\xi(1), \ldots, \xi(N)$ на $\xi(1), \ldots, \xi(p), \varepsilon(p+1), \ldots$ $\ldots, \varepsilon(N)$ якобиан равен единице и логарифм плотности вероятности, с точностью до постоянной, имеет вид (принимая во внимание, что матрица ковариации, а потому и обратная ей матрица величин $\xi(1), \ldots, \xi(N)$ является симметричной по обеим диа

$$
\begin{gathered}
\frac{1+\alpha_{1}^{2}+\ldots+\alpha_{p}^{2}}{2 \sigma_{\xi}^{2} A} \sum_{i=p+1}^{N-p} y_{i}^{2}+\frac{\alpha_{1}+\alpha_{1} \alpha_{2}+\ldots+\alpha_{p-1} \alpha_{p}}{\sigma_{\xi}^{2} A} \sum_{i=p+1}^{N-p+1} y_{i} y_{i-1}+\ldots \\
\cdots+\frac{\alpha_{p}}{\sigma_{\xi}^{2} A} \sum_{i=p+1}^{N} y_{i} y_{i-p}+\left(y_{1}^{2}+y_{N}^{2}\right) \frac{1}{2 \sigma_{\xi}^{2} A}+\left(y_{1} y_{2}+y_{N} y_{N-1}\right) \frac{\alpha_{1}}{\sigma_{\xi}^{2} A}+\ldots \\
\ldots+\left(y_{1} y_{p}+y_{N} y_{N-p+1}\right) \frac{\alpha_{p-1}}{\sigma_{\xi}^{2} A}+\left(y_{2}^{2}+y_{N-1}^{2}\right) \frac{1+\alpha_{1}^{2}}{2 \sigma_{\xi}^{2} A}+ \\
+\left(y_{2} y_{3}+y_{N-1} y_{N-2}\right) \frac{\alpha_{1}+\alpha_{1} \alpha_{2}}{\sigma_{\xi}^{2} A}+\ldots+\left(y_{2} y_{p}+y_{N-1} y_{N-p+1}\right) \frac{\alpha_{p-2}+\alpha_{1} \alpha_{p-1}}{\sigma_{\xi}^{2} A}+ \\
+\left(y_{p}^{2}+y_{N-p+1}^{2}\right) \frac{1+\alpha_{1}^{2}+\ldots+\alpha_{p-1}^{2}}{2 \cdot \sigma_{\xi}^{2} \cdot A} .
\end{gathered}
$$

Отсюда легко следует доказываемое утверждение. Таким же образом определяются достаточные статистики в случае, когда математическое ожидание неизвестно.

Следующий пример показывает, что в общем случае утверждение теоремы не имеет места. Рассмотрим стационарный гауссовский процесс

$$
\xi(t)=a_{0} \varepsilon(t)+a_{1} \varepsilon(t-1),
$$

где $\varepsilon(t)$ - независимые друг от друга гауссовские случайые величины. Покажем, чтов этом случае не существует функций от выборки $x_{1}, \ldots, x_{N}$, число которых было бы меньше $N$, таких, чтобы они образовывали достаточную статистику для $a_{0}, a_{1}, \sigma_{\xi}^{2}=$ $=\mathbf{M} \xi^{2}(t)$ (здесь предполагается, что $\mathbf{M} \xi(t)=0$ ). 
Из уравнения (5) следует, что

$$
\begin{aligned}
\sigma_{\xi}^{2}=M \xi^{2}(t)= & \left(a_{0}^{2}+a_{1}^{2}\right) \mathbf{M} \varepsilon^{2}(t), \rho=\frac{M \xi(t) \xi(t-1)}{\sigma_{\xi}^{2}}=\frac{a_{0} a_{1}}{a_{0}^{2}+a_{1}^{2}}, \\
& M \xi(t) \xi(t-\tau)=0, \text { если }|\tau|>1 .
\end{aligned}
$$

Совместная плотность вероятности величин $\xi(1), \ldots, \xi(N)$ имеет вид:

$$
p_{\xi(1), \ldots, \xi(N)}\left(y_{1}, \ldots, y_{N}\right)=\sigma_{\xi}^{-N} \cdot(2 \pi)^{-N / 2} \cdot\left|B_{N}\right|^{-1 / 2} \exp \left\{-\frac{1}{2 \sigma_{\xi}^{2}} \sum_{i, j=1}^{N} b_{i j}^{*} y_{i} y_{j}\right\},
$$

тде $\left|B_{N}\right|=\operatorname{det} B_{N}$ и $B_{N}^{-1}=\left\{b_{i j}^{*}\right\}_{1}^{N}$ - обратная матрица по отношению к корреляционной матрице $B_{N}$ процесса $\xi(t)$. Легко вычислить, что

$$
b_{i j}^{*}=(-1)^{j-i} \rho^{j-i}\left|B_{i-1}\right|\left|B_{N-j}\right| \frac{1}{\left|B_{N}\right|}
$$

при $i<j$ и $\left|B_{i}\right|=\frac{u_{1}^{i+1}-u_{2}^{i+1}}{u_{1}-u_{2}} \quad$ (обратная матрица предполагается симметричной по обеим диагоналям). Следует заметить, что $\left|B_{N}\right|$ удовлетворяет разностному уравнению $B_{N}|=| B_{N-1}\left|-\rho^{2}\right| B_{N-2} \mid$ и $u_{1}, u_{2}$ являются решениями уравнения

$$
u^{2}-u+p^{2}=0, \text { т. е. } u_{1}=\frac{1+\sqrt{1-4 \rho^{2}}}{2}, u_{2}=\frac{1-\sqrt{1-4 p^{2}}}{2} .
$$

Так как, далее, $b_{i N}^{*}(i=1, \ldots, N)$ независимы как функции от $\rho$, то становится ясным (см., например, Дынкин [2], $\S 2$ ), что не существует функций от выборки $x_{1},$. $\ldots, x_{N}$, число которых было бы меньше $N$ и которые образовывали бы достаточную статистику для $a_{0}, a_{1}, \sigma_{\xi}^{2}$.

Я считаю своим приятным долгом выразить глубокую благодарность А. Н. Колмогорову за постановку задачй и за внимание, оказанное мне при написании этой заметки.

$$
\begin{gathered}
\text { Поступила в редакиию } \\
21.4 .60
\end{gathered}
$$

\section{ЛITEPATYPA}

[1] Д ж. Л. Д у б, Вероятностные процессы, М., ИЛ, 1956.

[2] Е.Б. Дынки н, Необходимые и достаточные статистики для семейства распределения вероятностей, УМН, 6, 1 (1951), 68-90.

\section{SUFFICIENT STATISTICS OF STATIONARY GAUSSIAN PROCESSES}

\section{ARATO' (MOSCOW) \\ (Summary)}

We prove that for a stationary Gaussian process with spectral density (1) the number of sufficient statistics is $\frac{(p+1)(p+2)}{2}$. A simple example shows that in the general case the number of sufficient statistics increases with the number of observations. 